\title{
CONSIDERACIONES EN TORNO A CIERTAS PROPUESTAS NEOARISTOTELIZANTES O SOBRE LA DIFICULTAD DE ENCONTRAR TERCERAS VÍAS
}

\author{
Salvador MAS TORRES \\ Dpto. de Filosofia, UNED
}

Dentro de la amplia gama de "neoaristotelismos" que en la actualidad pueblan gran parte de la filosofia práctica pueden, quizá, distinguirse dos grandes grupos. De un lado estarían aquellas propuestas que han florecido sobre todo en el ámbito alemán, y que cabe considerar como anti-ilustradas o, incluso, pre-ilustradas; de otro lado, hay también intentos de conciliar la tradición aristotélica con ciertos valores puestos de relieve por la Ilustración y que, al parecer, son irrenunciables. En las páginas siguientes me ocuparé del segundo de estos grupos y, más en concreto, del intento de M. Nussbaum de conjugar el "esencialismo" y las "virtudes no relativas" con las ideas ilustradas de libertad y autonomía. ¿Es posible esta "tercera vía" que intenta reunir la idea de una moralidad sustantiva, en la tradición aristotélica, con el respeto, en la tradición ilustrada, de los diferentes deseos, necesidades y formas de vida de los individuos particulares?, ¿es factible, como cree Nussbaum, un aristotelismo no comunitarista, sino individualista?.

A) Será interesante detenerse por un momento en los argumentos que ofrece Aristóteles sobre la relación entre eudaimonia y tyche, pues aquí se encuentra una de las claves del aristotelismo de Nussbaum. Algunos piensan, dice Aristóteles, que la eudaimonia es totalmente invulnerable porque consiste en tener un buen estado o condición ética y porque esta condición es ella misma estable al margen de las circunstancias ${ }^{1}$. Aristóteles respondería que la eudaimonia no es un estado o condición, sino una actividad, precisamente la

1 Cfr. M. Nussbaum, The Fragility of Goodness, Cambridge, Cambridge Univ. Press, 1986, págs. 322 y ss. 
actividad del alma conforme a la virtud; y actuar conforme a la virtud (actuar "excelentemente") exige ciertas condiciones externas: del cuerpo, del contexto social, económicas; condiciones estas que están sometidas a los avatares de la fortuna. Aristóteles supone que el ser humano tiene una serie de capacidades, en el caso que ahora nos ocupa la capacidad de ser feliz, esto es, la capacidad de actuar conforme a la virtud; ahora bien, no basta con poseer la capacidad sin desplegarla realmente. Todo lo contrario: la "condición buena» de un agente encuentra su plenitud y su expresión más acabada en la actividad, y esta actividad sólo puede desarrollarse en el mundo. Pero entonces el mundo, por así decirlo, se toma su venganza e impone sus condiciones sobre el agente. Si éste ha sido "bien conducido por sus costumbres" y posee la "sabiduría práctica" podrá resistir los reveses de la fortuna; pero sólo hasta cierto punto. La vulnerabilidad, como muestra el caso extremo de Príamo, siempre es real ${ }^{2}$.

Al dejar paso de este modo a la fortuna Aristóteles diseña una concepción de la naturaleza humana en términos de capacidades, que está en la raiz de la propuesta neoaristotelizante de Nussbaum, pues que la fortuna juege un papel importante supone que la vida humana es, sobre todo, capacidad; capacidad que se desarrolla en el mundo y que el mundo puede frustar, por ejemplo: por mala suerte. Pero lo que en modo alguno resulta admisible es que estas capacidades no alcancen su pleno desarrollo porque los seres humanos vivan en un orden político que cercene de raíz su desenvolvimiento. Y esto resulta inaceptable porque la vida humana tiene "certain central defining features" ${ }^{3}$; Nussbaum no teme dar a esta teoría su verdadero nombre: esencialismo.

En un interesante trabajo titulado «Nature, Function and Capability: Aristoteles on Political Distribution" ${ }^{4}$ Nussbaum distingue dos tipos diferentes de capacidades: I-capacidades y E-capacidades. Las primeras quedan definidas como sigue: "Una persona es I-capaz de la función A en el tiempo t si y sólo si la persona está organizada en $t$ de tal modo que, cuando se le presentan las circunstancias adecuadas, esta persona puede elegir una acción A»; y las segundas: "Una persona es E-capaz de la función A en el tiempo t si y sólo si en $\mathrm{t}$ la persona es capaz de A y no hay circunstancias presentes que impidan o

2 Idem, pág. 340 y, en general, todo el capt. 12.

3 M. NusSBAUM, "Human Functioning and Social Justice: In Defense of Aristotelian Essentialismm, en Political Theory, 20, 2, 1992, pág. 205.

4 Reimpág. en G. E. MCCARTHY (ed.), Marx and Aristotle, Rowman \& Littlefield Publ., Maryland, 1992. 
dificulten el ejercicio de A». De esta forma, la tarea de todo orden político consiste en entrenar las I-capacidades en la juventud, mantenerlas en el adulto y, al mismo tiempo, crear y preservar las E-circunstancias en las que estas capacidades desarrolladas pueden tornarse activas. $O$, por decirlo con Aristóteles, "...corresponde al buen legislador considerar cómo la ciudad, el género humano y cualquier otra comunidad participará de la vida y de la felicidad que está a su alcance" 5 . Nussbaum señala que la misma idea vuelve a aparecer al comienzo de Pol. VII, 1, donde Aristóteles investiga cuál es el mejor orden político y sefiala que una teoría del buen orden político requiere y presupone una teoría de la vida humana buena: saber, en definitiva, qué vida es más preferible. Pero esto quiere decir que el criterio de bondad de un orden político es que los individuos que viven en él desarrollen bien su función, esto es, que sus I-capacidades y sus E-capacidades encuentren el marco adecuado para desenvolverse.

¿Quién es el sujeto de todo esto?, ¿a quién se dirige la tarea del legislador? Nussbaum contesta negativamente: de acuerdo con Aristóteles, mujeres y esclavos están excluidos, precisamente porque poseen capacidades distintas; y comenta a continuación que si pudiéramos separar el orden de los principios de sus injustas aplicaciones, en tal caso podríamos encontrar en este planteamiento aristotélico una base a partir de la cual cabría establecer la distribución de I y $\mathrm{E}$ capacidades ${ }^{6}$. Comentario este sumamente sorprendente, porque, en primer lugar, si hacemos esta separación a propósito de Aristóteles también podríamos hacerla, pongamos por caso, a propósito de la tiranía y decir: «si quitamos de la tiranía sus injustas aplicaciones...", lo cual, creo, repugnaría la sensibilidad política de Nussbaum. En segundo lugar, este comentario parece sugerir que existe un orden de los principios separado de sus aplicaciones, y esto es volver a caer en la separación entre teoría y praxis, lo cual -desde la lectura de Aristóteles que hace Nussbaum- es muy poco aristotélico. En cualquier caso, Nussbaum pasa por alto estas dificultades y concluye que el sujeto de la tarea del legislador lo es precisamente por tener por naturaleza (by nature) una capacidad menos desarrollada para realizar la acción, una capacidad tal que, dada la educación apropiada y los recursos externos necesarios, pueda llegar a su pleno desenvolvimiento. Nussbaum llama a esta capacidad «basiccapability»: "Una persona es B-capaz de la función A si y sólo si la persona

5 Pol. 1325 a $7-8$.

6 Cfr. «Nature, Function and...", pág. 191. 
tiene una constitución organizada para $\mathrm{A}$ de modo tal que es capaz de $\mathrm{A}$ dado el entrenamiento exigible, el tiempo y otras condiciones instrumentales necesarias". A lo cual habria que añadir, recordando lo ya dicho, que esta persona debe también tener suerte 0 , al menos, no tener la mala suerte de verse enfrentada a adversas circunstancias que le impidan el desarrollo de esta capacidad.

¿Cuáles son estas capacidades? ¿cómo confeccionar una lista de ellas? ¿y cómo hacerlo sin violar la idea ilustrada y liberal (a la que Nussbaum no quiere renunciar) de que los seres humanos deben ser libres para elegir autónomamente su propia concepción del bien? Nussbaum, buscando una "tercera via", establece dos exigencias que, en principio, parecen contrapuestas: hay que evitar un punto de vista externalista, que mire a la vida humana desde fuera de las condiciones y experiencias que configuran esta misma vida, como si la lista de capacidades pudiera establecerse a partir de hechos científicos acerca de nosotros mismos y que fueran axiológicamente neutrales. Pero esta exigencia no debe conducirnos, como sucede en otras perspectivas más o menos neoaristotalizantes ${ }^{7}$, al localismo comunitarista, pues junto a la exigencia de evitar el punto de vista externalista, hay también una exigencia de objetividad, en el sentido de que no cabe aceptar sin más ni más la información de los distintos individuos ${ }^{8}$, pues ésta puede estar sesgada, por ejemplo, por las malas circunstancias en que viven y se desarrollan los sujetos informantes: puede darse el caso de que en estas malas condiciones los sujetos ni siquiera sean capaces de concebir que existe determinada función. Nussbaum es muy clara a este respecto; y, asi, examinando las críticas de A. Sen al utilitarismo, señala que su perspectiva necesita una mayor radicalidad "....introduciendo una explicación objetivo-normativa del funcionamiento humano (buman functioning) y describiendo un procedimiento de evaluación objetiva con el que se pudiera determinar la contribución de esos funcionamientos (functionings) al bien de la vida humana" 9 .

B) A. Sen ha intentando investigar "una perspectiva particular sobre el bienestar y el beneficio en términos de la capacidad de una persona para realizar actos valiosos o alcanzar estados de ser valiosos" ${ }^{10}$. Este intento está próximo al

7 Cfr. «Non-Relative Virtues", en M. Nussbaum y A. SEN, (eds.) The Quality of Life, Clarendon Press, Oxford, 1993, págs. 243-244.

8 Cfr. "Nature, Function and...", pág. 198.

9 Idem, pág. 199.

10 "Capability and Well-Being”,en The Quality of..., pág. 30. 
aristotélico, pues implica que la cuestión distribucional debe ser llevada al contexto de las necesidades funcionales de cada individuo. Lo cual, a su vez, supone - también muy aristotélicamente- que hay que definir nuestra meta, nuestro telos, en términos de capacidades, y no en términos de nuestro funcionamiento actual.

En la raíz de las preocupaciones de Sen hay un problema económico: ¡en términos de qué medir el desarrollo? Una primera respuesta es la que ofrece el "Commodity Approach" y que consiste en identificar ciertos bienes o mercancĺas como intrínsicamente buenos; de esta forma, los ingresos per capita, el producto interior bruto o el crecimiento económico medido en términos de bienes y servicios (en tanto que cosas deseables en y por sí mismas) proporcionarían el criterio para medir el desarrollo. Esta forma del ver el problema cae en lo que Sen denomina "commodity fetishism", pues convierte en fines absolutos lo que son sólo medios instrumentales: en lugar de centrarse en la cuestión de lo que los individuos pueden hacer con estos bienes "11, el "Commodity Approach" los hipostasía convirtiéndolos en algo absoluto. Por otra parte, el "Commodity Approach" también vulnera el llamado «interpersonal variability argument» 12 : dadas las diferencias que existen entre los individuos el mismo bien puede ayudar a unos y empobrecer a otros, promover mucho la vida buena de unos y poco la de otros. El problema es aquí el mismo de antes: centrarse no en la persona que usa los bienes, sino en los mismos bienes, sin caer en la cuenta de que éstos varían en función de las personas.

El utilitarismo ha ofrecido otra respuesta a la pregunta por los términos en los que debe ser medido el desarrollo, a saber: en términos de bienestar (welfare), más exactamente, en los términos de la percepción subjetiva que los individuos tienen de su bienestar. El utilitarismo fue sometido a fuertes críticas por parte de Rawls, y Sen - como veremos- se inspira fuertemente en la crítica rawlsiana.

De acuerdo con Rawls de $A$ Theory of Justice, es rechazable el carácter meramente agregativo del utilitarismo, pues la maximalización del bienestar general puede ser perfectamente compatible con profundas desigualdades en

11 Cfr. A. SEN, Resources, Values and Development, Cambridge, Harvard Univ. Press, 1984, pág. 510.

12 Cfr. Resources, Values and..., pág. 511. 
su distribución. Y, por otra parte, no es obvio que sea precisamente el bienestar lo que debe de maximalizarse en primer lugar ${ }^{13}$, pues previamente habría que saber si los quanta de bienestar proporcionan un criterio adecuado para determinar si una sociedad es justa. Rawls, como es sabido, cree que no y, así, sustituye el criterio de bienestar por los bienes primarios. En escritos posteriores ${ }^{14}$ Rawls ha modificado su posición. Los bienes primarios, además de ser utilizados para derivar los principios de la justicia, han de ser justificados ellos mismos en tanto que son una exigencia de nuestra concepción de los seres humanos como "personas morales" libres e iguales. Las personas morales, señala Rawls ${ }^{15}$, se caracterizan, en primer lugar, por tener un sentido de la justicia, esto es, por ser capaces de entender, aplicar y actuar de acuerdo y desde principios de justicia; en segundo lugar, por la capacidad de formar, revisar y perseguir racionalmente una concepción del bien. En este contexto de personas morales los bienes primarios son, simplemente, condiciones de posibilidad para la igualdad y la libertad de los ciudadanos. Esta mayor importancia que Rawls asigna ahora a los bienes primarios implica (no podía ser de otra manera) una ampliación del conjunto de bienes que, en efecto, han de ser considerados primarios ${ }^{16}$.

No olvidemos el problema que nos ha llevado a realizar esta disgresión acerca de la crítica de Rawls al utilitarismo: ¡en términos de qué medir el desarrollo?. No en términos de mercancías, tampoco en términos de la percepción subjetiva del "bienestar». ¿Será posible hacerlo en términos de «bienes primarios»? Según Sen tampoco esta es buena solución, pues Rawls, huyendo del utilitarismo, ha caldo en el "commodity fetishism": sigue considerando los bienes en sí mismos y no lo que con los bienes hacen los seres humanos ${ }^{17}$. Para Rawls los bienes primarios nos dicen los que seres humanos desean y consideran su "rational advantage», pero sólo en caso — señala Sen- de que los individuos fueran similares. Rawls, pues, ha puesto de manifiesto la "inter-end variation" ${ }^{18}$ que indica que diferentes individuos tienen diferentes concepciones del

13 Cfr. A Theory of Justice, Cambridge, Harvard Univ. Press, 1971, pág. 91.

14 Cfr. "The Priority of Right and Ideas of the Good», en Philosophy and Public Affairs, 17, 1988, págs. 251-276; tb. "Kantian Constructivism in Moral Theory: The Dewey Lectures 1980m, en Journal of Philosophy, 77, 9, 1980, págs. 515-572.

15 «Kantian Constructivism...", pág. 535.

16 Cfr. *The priority of...* pág. 257.

17 Cfr. A. SEN, "Equality of What?", en S. MCMURRIN (ed.), Tanner Lectures on Human Values I. Cambridge, Cambridge Univ. Press, 1980, pág. 218. 
bien, pero ha pasado por alto que «las variaciones relacionadas con el sexo, la edad, la dotación genética y con otros muchos rasgos nos dan poderes desiguales para construir la libertad en nuestras vidas, incluso cuando tenemos los mismos conjuntos de bienes" ${ }^{19}$.

C) ¿No se podría considerar la lista de bienes primarios como aquellos "...central defining features" de los que hablaba Nussbaum? En alguna medida sí, pero esto supondría ir mucho más allá de lo que Rawls aceptaría. Rawls, en efecto, rechaza como utópica cualquier teoría moral que incluya una concepción "de lo que tiene un valor último en la vida humana" ${ }^{20}$ : lo que denomina «a comprehensive theory». Frente a este tipo de teorías, la concepción política de la justicia debe entenderse como un "overlapping consensus" de y entre ciudadanos libres e iguales. Por otra parte, Rawls rechaza también cualquier teoría general que se aplique a cualesquiera aspectos de la vida en general, y no sólo - como es el caso de su propuesta- a la esfera pública: el «overlapping consensus" sólo se dice de los bienes instrumentales y de los principios distributivos relevantes en la esfera pública ${ }^{21}$. Sin embargo, sólo desde una teoría "general" y "comprehensiva" puede hacerse justicia al argumento de la variacion interindividual. Rawls, como ya se ha sefialado, pasa por alto la variación interindividual en la relación entre bienes primarios y la libertad para perseguir fines, y, así, la igualdad entendida al modo de los bienes primarios puede solaparse con profundas desigualdades en las libertades reales disfrutadas por diferentes personas ${ }^{22}$.

Rawls, por su parte, sostiene que este argumento está viciado de entrada, pues presupone que su teoría es lo que no es, a saber: una teoría moral general y comprehensiva. La respuesta es paradójica ya que, en el fondo, viene a dar la razón a Sen: desde una teoría como la de Rawls (que es una teoría no general y no comprehensiva) no se puede hacer justicia al argumento de la variación interindividual. Y, sin embargo, este argumento no es en modo alguno

18 A. SEN, "Justice: Means versus Freedomsw, en Philosopby and Public Affairs, 1990, 19 (2), pág. 120.

19 Idem pág. 121.

20 "The Priority of...", pág. 252.

21 Lo correcto (right) sigue detentando la prioridad sobre el bien, en la medida en que son los principios de la justicia los encargados de poner límites a las diversas y, en ocasiones, enfrentadas concepciones del bien: "the principles of justice se limits to permissible ways of life" ("The Priority of...", pág. 251).

${ }^{22}$ Cfr. A. SEN, «Justice...", pág. 115. 
irrelevante, pues pone de manifiesto que una libertad entendida de forma meramente negativa como la ausencia de restricciones que una persona puede ejercer sobre otra puede ser profundamente injusta con respecto a las variaciones interindividuales. La libertad positiva exige también otro tipo de restricciones, por ejemplo, de la pobreza y de la ignorancia, esto es, exige dar prioridad al concepto de bien, para lo cual parece obligado abrirse a una teoría general y comprehensiva. Rawls, pues, tendría que haber planteado una teorla "más comprehensiva", pero no lo hace -quizá por una vergüenza epistemológica de origen más o menos remotamente analítico. Como dice Nussbaum: "Rawls theory, then, is too thin" ${ }^{23}$; demasiado tenue, en efecto, para lo que se propone, pues lo coherente, señala Nussbaum, habría sido concebir los bienes primarios como siendo parte ellos mismos de una concepción del bien. De hecho, esto es lo que hace implícitamente Rawls, pues de lo contrario, o sea, sin asumir (si bien soterradamente) una concepción comprehensiva de lo que es el bien para una vida humana, no podría defender su propia teoría de los bienes primarios.

Nussbaum exige de Rawls lo mismo que exigía de Sen: una mayor radicalidad. En el caso de Rawls esta exigencia adoptaría la forma de hacer explícito algo que en alguna medida ya opera implicitamente, pues concebir los bienes primarios como siendo parte ellos mismos de una concepción del bien obligaría a: 1) introducir una explicación objetivo-normativa del funcionamiento humano y 2) describir un procedimiento de evaluación objetiva con el que se pudiera evaluar la contribución de ese funcionamiento al bien de la vida humana ${ }^{24}$.

En respuesta a Nussbaum, $\operatorname{Sen}^{25}$ reconoce que aunque hay grandes similitudes entre el "capability approach" y la perspectiva aristotélica, no puede ocultarse que existen también "algunas diferencia substanciales»: "Aristóteles creía, como indica Nussbaum, que sólo hay una lista de funciones que constituyan de hecho una vida humana buena" ${ }^{26}$. No es que el "capability approach" niegue esto, lo que sucede - piensa Sen- es que en modo alguno lo exige. En efecto, Sen ha utilizado el "capability approach" para argumentar que mientras que las exigencias de mercancías y bienes pueden variar mucho

\footnotetext{
23 .Nature, Function and...", pág. 180.

24 "Nature, Function and...n, pág. 199.

25 "Capability and Well-Being", en The Quality of Life, págs. 46 y ss.

26 Idem pág. 46. Sen se refiere al trabajo de Nussbaum «Nature, Function and...*.
} 
de una comunidad a otra, hay mucha menos variación en las capacidades que se quieren adquirir a través del uso de esas mercancías y bienes ${ }^{27}$. Es cierto que por este camino se podría llegar a la tesis aristotélica de un conjunto de «virtudes no relativas» ${ }^{28}$, pero "the Aristotelian claim of uniqueness go much further" ${ }^{29}$ de lo que Sen pretende. Por una parte, Sen reconoce que el planteamiento aristotélico de Nussbaum sería una buena forma de eliminar el carácter incompleto del "Capability Approach", pero por otra parte sefiala que sus dificultades con la perspectiva aristotélica nacen de que ésta está excesivamente especificada: no deja espacio ni para la libertad ni para la autonomía ${ }^{30}$. Y es que el carácter incompleto del "Capability Approach", lejos de ser un inconveniente, constituye una ventaja, a saber, la de hacerlo consistente y combinable con un amplio conjunto de teorías sustantivas.

Lo que ocurre es que esto último no es algo propio y específico de la teoría de Sen. También la de Rawls, por ejemplo, es consistente y compatible con un amplio conjunto de teorías sustantivas, siempre que éstas acepten las libertades contenidas en el primer principio de la justicia y defiendan una política socioeconómica dirigida a propiciar la igualdad de oportunidades y la preservación de un mínimo vital para todos los sectores sociales ${ }^{31}$. Por otra parte, Sen insiste en que el verdadero interés de su perspectiva reside en su "cuttingpower": el "capability-approach" niega que podamos tomar pie en las mercancías, en la satisfacción de deseos, en los recursos como tales, en los bienes primarios. En esta perspectiva crítica reside su mayor interés. $\mathrm{El}$ problema es entonces cómo pasar de la parte crítico-negativa a la parte positivo-constructiva, lo cual parece muy difícil sin una definición esencialista de la noción de "capacidad" (es decir, parece difícil dar este salto manteniendo el carácter incompleto de la teoria). Sen se niega a todo esencialismo, poco más o menos por las mismas razones por las que Rawls se negaba a una teorfa general y comprehensiva: ambos quieren ser liberales. ¿Cómo, entonces, definir las capacidades dentro de un marco semejante?.

D) Las capacidades se definen derivadamente a partir de las "functionings" y éstas, a su vez, representan partes del estado (state) de una persona.

\footnotetext{
Cfr. Idem pág. 47.

Cfr. M. NusSBauM, „Non-Relative Virtues...», págs. 242-269.

"Capability and...", pág. 47.

Idem pág. 47.
} 
De esta forma, la capacidad de una persona refleja las combinaciones alternativas de "functionings" que la persona puede realizar. Sen reconoce que en esta perspectiva la noción más primitiva no es la de "capacidad», sino precisamente la de "functioning" ${ }^{32}$, pues si la realización de una función (functioning achievement) es un punto en el espacio de las "functionings", la capacidad es un conjunto (set) de tales puntos. ¿Por qué, entonces, si el concepto más básico es el de "realización de una función», la teoría de Sen, como su propio nombre indica, considera a las capacidades como la base informacional primaria? ¿De dónde nace la relevancia de la noción "conjunto de capacidades" para el análisis del well-being?. El problema, en definitiva, es saber en términos de qué hacer el análisis, ¿en términos de "capacidades" o en términos de "estados"?. Y tras esta pregunta, como muy bien ha visto el mismo Sen, lo que se esconde es «a substantive issue» ${ }^{33}$, pues la preferencia por parte de Sen del análisis en términos de capacidades nace de que este análisis deja espacio a la libertad ${ }^{34}$, cosa que no ocurriría (o, al menos, no de forma tan clara) en un análisis en términos de estados. Si se parte de que el ser humano en tanto que tal (y no, por ejemplo, en tanto que flautista o zapatero) tiene un "estado" que lo define como ser humano, en tal caso es este mismo estado el que limita el número de funciones y de capacidades que cabe considerar como especificamente humanos, y la capacidad de elegir libre y autónomamente unos bienes y no otros queda también predeterminada por la noción de "state of a person". Habría entonces que decir, con Aristóteles, que aunque todo lo que un ser humano escoge es "su bien", cuando lo que escoge son "cosas malas", entonces este bien sólo lo es en apariencia. La contrapartida de esta (digámoslo así) pérdida de libertad es que un análisis un términos de "estados" facilita enormemente la cuestión de la distribución igualitaria de los bienes ${ }^{35}$, precisamente porque de la igualdad de estado se sigue directamente la necesidad de una distribución igual de los bienes y de la desigualdad de estado se sigue no menos directamente la desigualdad en la distribución de bienes.

31 Cfr. F. VALlESPIN, “El neocontractualismo: John Rawls», en V. CAMPS (ed.) Historia de la Etica, vol. III, Madrid, Crítica, 1989, pág. 590.

32 Cfr. kCapability and...", pág. 38.

33 "Capability and...", pág. 43.

34 Idem pág. 45.

35 Recordemos, sin embargo, que la cuestión distributiva era el eje de una de las críticas que Rawls dirige al utilitarismo. 
Rawls ${ }^{36}$ ha distinguido entre teorías políticas liberales y no liberales. Para las primeras, el propósito del Estado es permitir que cada individuo persiga su propia concepción del bien. En las teorías no liberales, por el contrario, se considera establecida una determinada concepción del bien, y la tarea del Estado es realizar esta concepción. La teoría aristotélica sería un ejemplo; hay, en efecto, una determinada concepción del bien: vivir de acuerdo con la virtud, y la tarea del buen legislador consiste, consiguientemente, en educar a los ciudadanos para que puedan llevar una vida de acuerdo con la virtud. ¿Cómo se puede ser a la vez liberal y aristotélico?. Evidentemente, de ninguna manera si uno es liberal, por ejemplo, al modo de Kant, que pensaba que la tarea del Estado era proteger derechos y libertades, y no tanto facilitar la consecución de una vida buena. Cabe, sin embargo, una vía intermedia ${ }^{37}$, que coincidiría con las teorías no liberales en afirmar que la tarea del Estado consiste en hacer a los ciudadanos capaces de llevar a cabo una vida buena, pero que a diferencia de estas teorlas defiende que no hay establecida una única concepción del bien, sino que cada individuo debe construir y perseguir libre y autónomamente su propia concepción. Esta posición intermedia trataría de hacer compatible la posición aristotélica con los supuestos del individualismo, tanto ético como epistemológico, y, así, vía Aristóteles resolver el problema de la distribución igual de bienes, y vía individualismo hacer justicia a la exigencia de libertad. Tal es el diffcil empeno en el que está embarcada M. Nussbaum: ¡cómo conjugar su esencialismo y su apelación a unas "virtudes no relativas" con las demandas de libertad y autonomía?

E) En "Non-Relative Virtues..." se plantea Nussbaum si es posible llegar a una lista de virtudes que no sea un simple reflejo de valores y tradiciones meramente locales. Suele decirse que este es justamente el planteamiento de Aristóteles, el cual se habría limitado a enumerar e individualizar las virtudes (y vicios) operantes en la sociedad de su época. Pero esta lectura, senala Nussbaum, es errónea. Lo que realmente hace Aristóteles es aislar una esfera de la experiencia humana que esté presente en toda vida humana, y en la que todo ser humano tenga que elegir y actuar; y en un segundo momento se pregunta qué es elegir y actuar bien (o mal) dentro de cada una de estas esferas.

36 "Social Unity and Primary Goods", en A. SEN y B. Williams (ed.) Utilitarianism and Beyond, Cambridge Univ. Press, 1982, págs. 159-185.

37 Cfr. el comentario de CH. M. KORSGaARD a los trabajos de COHEN y SEN, en The Quality of Life, pág. 56. 
A partir de esta investigación obtiene, en primer lugar, una "thin account" de cada una de las virtudes: saber en qué consiste la disposición estable para actuar y elegir apropiadamente dentro de cada una de estas esferas. Pero puede ser que existan diversas especificaciones de lo que es, en cada caso, actuar bien. En esta situación, Aristóteles ofrece en cada caso una especificación más concreta, dando lugar así, en segundo lugar, a una «full or thick definition of the virtue" ${ }^{38}$. El nervio de esta elucidación de cada una de las virtudes lo constituye una investigación de carácter lingüístico. En efecto, Aristóteles - tanto en sus investigaciones prácticas como en las científicas- siempre comienza con una experiencia, bien sea de uno mismo bien sea de algún miembro de nuestra comunidad linguística, y a partir de estas experiencias radicales (grounding experiences) hace su entrada una palabra que indica cuál es el contenido de esta experiencia ${ }^{39}$. En Analíticos Segundos (93 a 21 y ss.) Aristóteles pone el ejemplo del trueno: de lo que se trata es de conseguir una definición, esto es, de especificar el sentido de la palabra "trueno" a partir de la experiencia ingenua y primera de oir un ruido en las nubes. En cuestiones de filosoffa práctica el proceso sería el mismo, y así «...podemos comprender el progreso en ética, como el progreso en la investigación científica, como un progreso para encontrar la correcta especificación satisfactoria de una virtud, especificada por su definición débil o nominal» ${ }^{40}$.

La misma Nussbaum señala tres posibles objeciones a esta propuesta ${ }^{41}$. En primer lugar, sucede que aunque se admita que Aristóteles ha tenido éxito en su empeño de singularizar el problema señalando y describiendo correctamente las esferas de experiencia sobre las que se levantan cada una de las virtudes, ello no implica que quepa también dar una respuesta singular al problema, pues bien pudiera ser que sobre una misma y singular área de experiencia no se levantara una única virtud, sino una pluralidad de narrativas que ofreciera, cada una de ellas, una definición fuerte de la virtud en consonancia con las experiencias y tradiciones respectivas de cada grupo humano. En segundo lugar, Aristóteles parece pensar que las experiencias que están en la raíz de sus investigaciones son experiencias primitivas, dadas y al margen de las variaciones culturales que cabe encontrar en la pluralidad de concepciones normativas

\footnotetext{
38 «Non-Relative Virtues...., pág. 245.

39 Cfr. M. Nussbaum, The Fragility of..., capt. 8.

40 "Non-Relative Virtues...., pág. 248.

41 Cfr. idem págs. 251 y ss.
} 
sobre la virtud. Lo cual es una ingenuidad: no existe tal "ojo inocente" que capte «lo dado" inmediatamente y al margen de toda interpetación. En tercer lugar, cabe radicalizar esta segunda objeción y señalar no sólo la diversidad y pluralidad de las experiencias radicales, sino también la posibilidad de que existan formas de vida que carezcan de tales experiencias o, al menos, de algunas de ellas. Por otra parte, puede también pensarse que tales experiencias tienen un carácter «ideológico", en el sentido clásico marxiano de la palabra: obscurecimiento y deformación de la realidad con el objeto de presentar como virtudes universales lo que son sólo virtudes de un grupo humano determinado.

Las tres objeciones son modulaciones (en distinto tono y con diferente nivel de radicalidad) de las críticas a la posición esencialista. Y las tres insisten en acentuar tesis relativistas. La tarea de Nussbaum es, pues, difícil: tiene que hacer justicia a estos desafíos relativistas (pues no puede negar que exista pluralidad y diversidad, ni aceptar que la pluralidad y la diversidad tengan que ser vistas como aberración y desvario), pero sin caer ni el relativismo ni en la "metafísica». Nos encontramos, pues, con un no-relativismo, cuanto menos, alejado de lo que suele entenderse por no-relativismo. Pues es un no-relativismo que ante la pregunta "¿qué es X?" acepta diversas especificaciones (una respuesta no singular, sino plural), que es "context-sensitive" (lo cual, añade Nussbaum sin mayor explicación, "no implica que sea correcta sólo relativamente a, o dentro de, un contexto limitado" ( $\left(\zeta\right.$ ?) " ${ }^{42}$ ) y que está siempre abierto a la revisión a la luz de las nuevas circunstacias. ¿Cómo conjugar este peculiar no-relativismo con el esencialismo?.

F) Las versiones más tradicionales del esencialismo han sido sometidas a fuertes críticas desde las más diversas perspectivas ${ }^{43}$. Por una parte, desde un punto de vista epistemológico, se ha criticado su vinculación con un realismo metafísico que resulta insostenible. El realismo metafísico, por decirlo con una simplificación quizá abusiva, sostiene que el mundo es independiente de las facultades cognoscitivas de los seres humanos, de suerte que una descripción del mundo será verdadera si se corresponde con esa estructura que es totalmente independiente del sujeto, y será falsa en caso contrario. En la medida en que esta tesis epistemológica se derrumba parece que tendría también que derrumbarse la visión esencialista, pues si el acceso al mundo está filtrado por

42 Idem, pág. 257.

43 Cfr. «Human Functioning and...n, págs. 202-246. 
toda una serie de instancias mediadoras no se comprende entonces cómo se podría captar en sí misma, al margen de la historia y de toda interpretación, esa supuesta esencia del ser humano. Por otra parte, y ahora desde el punto de vista de la filosofia práctica, se ha dicho que el esencialismo deja de lado las diferencias históricas y culturales y hace imposible toda idea de autonomía, pues en nombre de una supuesta e inmutable esencia del ser humano puede llegarse a anatemizar como "anti-natural" todo lo que no se corresponda con tal esencia, $y$ asímismo en su nombre puede también negarse el derecho que tienen los seres humanos a elegir qué es aquéllo que consideran decisivo a la hora de construir su plan de vida. En tercer lugar, el esencialismo también ha sido criticado por su "prejudicial application": el mismo Aristóteles cae en este error, pues el esencialismo, en sus manos, le lleva a excluir a mujeres y esclavos de la condición de seres humanos en el sentido más pleno y más acabado de la palabra.

La teoría que presenta Nussbaum pretende superar todas estas dificultades y, sin embargo, seguir siendo esencialista; ella misma se refiere a su posición como «esencialismo empírico fundamentado históricamente" y propone denominarlo, buscando intencionadamente la analogía con las tesis epistemológicas de Putnam, "esencialismo interno" "As. Así pues, aunque acepta que muchas concepciones esencialistas son susceptibles de ser críticadas en los términos antes mencionados $u$ otros parecidos, señala que ello no implica que todos los esencialismos tengan necesariamente que caer en las mismas dificultades ${ }^{45}$.

La estrategia de Nussbaum consiste en identificar un grupo de funciones que sean especialmente relevantes en la vida humana, pues entonces se estará en condiciones de preguntar por la relación entre las instituciones sociales y politicas y dichas funciones, y se podrán formular juicios evaluativos en función de la circunstancia de si las instituciones favorecen el pleno desarrollo de tales funciones o si, por el contrario, lo impiden. En contraste con las tesis de Rawls, Nussbaum denomina a su propuesta esencialista "thick vague theory of the goodn: a diferencia de la de Rawls, la concepción de Nussbaum «tiene que ver con los fines y con el perfil general y el contenido de una forma humana de vida" "46. Y es deliberadamente "vaga" para admitir múltiples especificaciones de

\footnotetext{
44 Cfr. Idem, pág. 208.

45 Cfr. Idem pág. 214.

46 Idem pág. 215.
} 
acuerdo con las variadas concepciones locales y personales. El problema, entonces, es de dónde saca Nussbaum esta lista de "capacidades funcionales humanas básicas", sobre todo teniendo en cuenta que rechaza, por metafísica, la posibilidad de derivarla a partir de alguna fuente externa a la realidad histórica y social de los mismos seres humanos. Nussbaum en modo alguno está interesada en una ontología de los valores tipo, pongamos por caso, Scheler. Ni tampoco puede aceptar un reduccionismo naturalista de corte más o menos biologicista. ¿Qué solución, pues, es la que propone?

Nussbaum es sumamente ambigua a propósito de esta importante cuestión. Así, en "Human Functioning...." sefiala que hay que proceder tomando en consideración una gran variedad del conjunto de las autocomprensiones que los seres humanos tienen y han tenido de si mismos a lo largo del tiempo y en diferentes espacios; entonces "La gran convergencia entre culturas que se da en tales reconocimientos o negativas de reconocimientos nos da alguna razón para ser optimistas, o sea, para pensar que, si procedemos de esta forma, usando nuestra imaginación, tendremos al final una teorla que no es la mera proyección de nuestras propias costumbres, sino que también es plenamente internacional y un punto de partida para la armonía intercultural» ${ }^{47}$. El argumento es claramente vicioso, pues presupone como probado aquello que habria que probar, a saber: que existe una gran convergencia entre las diversas culturas. Por otra parte, a la hora de enfrentarse con las autocomprensiones de culturas muy lejanas a la nuestra (con el objeto de ver si, a fin de cuentas, hay no hay tal "gran convergencia») surgen una serie de problemas hermenéuticos que no se pueden despachar simplemente "usando nuestra imaginación", pues puestos a imaginar cabe imaginar cualquier cosa, incluso cosas contrapuestas, ¿y cómo, entonces, decidir entre alternativas excluyentes?. Aunque se acepte que el no-relativismo tiene que ser vago, la vaguedad tiene que tener un límite, pues de lo contrario sería imposible de todo punto conjurar el peligro relativista.

$Y$ con esto entramos en la segunda objección que la misma Nussbaum reconoce que puede hacerse a su posición, pues del hecho de que no exista un "ojo inocente" (lo cual, obviamente, acepta) no se sigue que todas las interpretaciones del mundo posibles sean igualmente válidas; hay que poner ciertos límites, ya que de lo contrario caeríamos en la paradoja de un no-relativismo relativista: "Ciertos formas en las que la gente ve el mundo pueden ser criticadas

47 Idem pág. 216. 
exactamente como Aristóteles las criticó: como estúpidas, perniciosas y falsas. Los patrones usados en tal crítica deben venir de dentro de la vida humana ${ }^{48}$. Con lo cual regresamos a la cuestión del esencialismo, pues estos patrones usados en la crítica y que deben venir desde dentro de la misma vida humana se solapan exactamente con la lista de capacidades funcionales humanas básicas, ya que son estas capacidades las que, por encima de las diferencias en la concepción de las experiencias radicales, nos permiten que reconozcamos las experiencias de otras culturas como semejantes a las nuestras. La idea es sugerente, pero muy imprecisa, pues no se puede olvidar que el esencialismo supuestamente limitador es también, él mismo, "vago" y que, en consecuencia, también limitaría "vagamente» la multitud de interpretaciones posibles. Y entre esta multitud de interpretaciones posibles está aquella que recoge la tercera objección, a saber, que existan formas de vida que carezcan de las experiencias radicales entendidas al modo aristotélico o que consideren ideológicas tales experiencias. ¿Qué pasa aquí, ¿¿tiene la vaguedad que ser tan amplia como para seguir considerando seres humanos a aquellos grupos que carezcan de tales experiencias?, ¿đónde y cómo poner límites?

En cualquier caso, todas estas dificultades no tienen mucha importancia si con la lista de "capacidades funcionales humanas básicas" lo único que se pretende, y así parece sugerirlo la misma Nussbaum, es conseguir "a very wide consensus" ${ }^{49}$ que posibilite la crítica política. Pero si este es el caso, no se entiende entonces qué necesidad hay de hablar de "esencialismo": de hecho, si lo único que aquí está en juego es de llegar a tal consenso, no estaríamos muy lejos del "overlapping consensus" de Rawls. Es cierto que desde este peculiar esencialismo cabe muy bien responder a las críticas anti-esencialistas que la misma Nussbaum recoge y de las que me hacía eco unas páginas más arriba. Pero cabe sospechar que el esencialismo de Nussbaum es tan vago, tan respetuoso de la libertad y de la autonomía y tan susceptible de ser aplicado en una dirección o en otra, que, a fin de cuentas, ha dejado de ser esencialismo; o quizá puede ser que Nussbaum esté empleando la palabra «esencialismo" en un sentido novedoso... pero que en modo alguno es aristotélico.

En dos palabras: el esencialismo de Nussbaum es "vago" por las mismas razones por las que el "Capability Approach" de Sen era uincompleto". En

48 "Non-Relative Virtues...", págs. 260-261.

49 "Human Functioning and...", pág. 223. 
principio, nada hay que objetar a ello, sobre todo una vez que se supera el ligero malestar producido por utilizar una palabra («esencialismo») en un sentido muy diferente del habitual. Pero si este es el caso, no se comprenden entonces las críticas que Nussbaum dirige a Sen: también cabría exigir de la primera una mayor radicalidad (y por las mismas razones por las que ella se la exige a Sen). Pero entonces desaparecería la vaguedad y, en consecuencia, ya no sería tan fácil responder a las críticas anti-esencialistas. Habría, en efecto, que mantener un esencialismo fuerte -como, por ejemplo, hacen los aristorelismos antiilustrados-; pero de esta forma recaeríamos en las posiciones comunitaristas que Nussbaum quiere evitar y desaparecerían también las virtualidades como "tercera vía" de su propuesta neoaristotelizante

G) Quizá urgida por estas y otras dificultades semejantes, Nussbaum, en su último libro ${ }^{50}$, toma distancia frente a las propuestas aristotélicas, más exactamente, pone de manifiesto las profundas deficiencias de estas propuestas y parece decantarse en favor de un "modelo terapéutico" para la reflexión práctica, en un tradición no tanto aristotélica cuanto helenista y, especialmente, estoica. Cabe aclarar esta concepción de la filosofia práctica, que se apoya en la analogía médica y que también se presenta como «tercera via», comparándola con otras dos concepciones: la platónica y la que toma pie en la creencia ordinaria; esta última concepción, señala Nussbaum, ha sido remitida a Aristóteles y, en efecto, tiene elementos en común con la posición aristotélica. En cualquier caso, no son Platón o Aristóteles considerados en sí mismos los que aquí están en juego, puesto que estas dos concepciones siguen estando presentes en la reflexión contemporánea sobre cuestiones de filosofia práctica ${ }^{51}$.

La concepción platónica considera que las normas éticas son lo que son independientemente de los deseos y de las formas de vida de los seres humanos y, en consecuencia, sostiene que es puramente contingente la relación entre nuestros intereses y deseos y el bien verdadero. Esta concepción puede adoptar dos variantes: religiosa o científica. En el primer caso, las normas éticas son independientes de los seres humanos en la medida en que se identifican, por ejemplo, con los designios divinos; en el segundo, de acuerdo con Nussbaum, la investigación ética consiste en el descubrimiento de verdades sobre normas y valores, verdades totalmente independientes de lo que somos,

so The Therapy of Desire, Princeton, Princeton Univ. Press, 1994.

51 Cfr. Idem, pág. 16. 
queremos o hacemos. $Y$ en nota a pie de página aniade: «Veáse Rawls para una explicación de esta perspectivan ${ }^{52}$. Evidentemente, Rawls no aceptaría esta caracterización; pero dejemos de momento a un lado esta cuestión y veamos ahora en qué medida la concepción terapéutica subvierte la idea fundamental de la concepción platónica, esto es, la idea de la radical independencia del bien verdadero respecto de los deseos y necesidades de los seres humanos. Y la subvierte porque el fin del médico, que es ayudar, no puede ser alcanzado independientemente del sentido que tiene el paciente de lo mejor y lo peor: la salud, escribe Nussbaum, no existe en un cielo platónico, totalmente al margen de la gente y de sus vidas. Conclusión: la salud no es un concepto absoluto, sino relativo.

De lo dicho hasta el momento parece desprenderse que la salud es relativa a la percepción subjetiva que de ella tienen los individuos y las culturas. Imaginemos ahora una persona mal alimentada y que, precisamente por su mala nutrición, tiene una percepción distorsionada de lo que es la salud: si se la alimenta adecuadamente reconocerá, comparando su estado presente con el pasado, que antes estaba enferma. Pero de esta forma se desplaza el punto de referencia de la relatividad de la salud, que ahora no es tanto la percepción subjetiva de la salud, cuanto el saber que tiene el médico de lo que es salud, pues es el médico el que sabe que, en condiciones de mala alimentación, la percepción de la propia salud no puede ser correcta, y es el que prescribe la dieta adecuada: el saber del médico es condición de posibilidad de la modificación de la percepción de la propia salud. Más llamativo es el desplazamiento en el siguiente caso: hay sociedades, como ha mostrado A. Sen, en las que la percepción de la propia salud está sesgada por el patrón de dominación género/sexo, pues sucede que, en virtud de la educación y de la información poseida, varones y mujeres igualmente sanos o enfermos responden diferentemente a la pregunta por su salud. A este respecto, y después de reconocer que en este caso es más difícil que el sujeto reconozca "the evidence of disease", Nussbaum sefiala que la combinación de la evidencia médica con la presentación de comparaciones con otras culturas o con otros individuos de la misma cultura y, si es posible, con las comparaciones posibilitadas por el cambio en la propia condición del paciente, puede llevar a un juicio crítico frente a la tradición "and supportive of the doctor's hypothesis" "53. Totalmente de acuerdo, sólo que en este caso «the doctor's hypothesis" ha dejado de ser una hipótesis

\$2 Idem, pág. 18, n. 11.

53 Idem, págs. 20-21. 
y se ha convertido en una tesis, o sea, en un criterio objetivo de salud independiente de los deseos y formas de vida del paciente. Huyendo del relativismo se ha caído en el platonismo. Y también totalmente de acuerdo con el paralelismo que establece Nussbaum entre la salud del cuerpo y la vida buena desde un punto de vista ético: "Así como una condición intolerablemente tullida del cuerpo no puede ser lo que es la salud, del mismo modo una forma de vida inaceptablemente monótona, empobrecida o dolorosa no puede ser lo que es la vida bumana buenas ${ }^{54}$. Ahora bien, es el médico el que establece cual es la condición tullida del cuerpo, ¿pero quién hace lo propio con la vida humana (supuesta o realmente) inaceptable?

No conviene olvidar que también en Platón aparece la analogía médica. En el Gorgias, por ejemplo, tomando a la medicina como modelo, se define al verdadero "arte político" como therapeia del alma; la medicina puede curar porque conoce previamente la naturaleza de aquello que cura y, en consecuencia, puede decir la causa de lo que hace 55 . De igual modo, el verdadero "arte politico" podrá curar su objeto porque previamente lo conoce, es decir, conoce la naturaleza del alma; sabe, por tanto, cuáles son sus enfermedades y cómo reconducirla al estado de salud. Si como cree Platón hubiera médicos del alma que conocieran perfectamente cuál es el verdadero estado de salud de la polis sería muy fácil evitar que la analogía médica condujera al relativismo. Pero Nussbaum está justamente criticando la concepción platónica.

Sin embargo, a pesar de esta posición anti-platónica Nussbaum cree justificado seguir hablando de "verdad" a propósito de estas cuestiones. Y si acusa a Rawls de platónico es porque considera que éste piensa que en el contexto de la filosofia práctica no tiene cabida el concepto de verdad. Ciertamente, Rawls ha sostenido que la investigación ética es esencialmente una cuestión práctica y que no consiste en el descubrimiento de algo que está fijado con independencia de nosotros; sin embargo, impresionado por la distinción (según Nussbaum platónica) entre ética y física, mantiene que la noción de verdad sólo es apropiada para aquellas investigaciones de la naturaleza de una realidad independiente de nosotros; y como la ética no tiene tal meta independiente, no puede reclamar el uso de la noción de verdad ${ }^{56}$. La crítica es

Idem, pág. 22.

Cfr. Gorgias, 501 a.

s6 The Therapy of..., pág. 22. 
apresurada e injusta, pues de que Rawls se muestre partidario de la sobriedad epistemológica y metodológica y se aleje conscientemente de las grandes construcciones téricas (como, en efecto, hace ${ }^{57}$ ) no parece muy adecuado concluir que es un platónico. Caracterizar al platonismo como la tesis que sostiene que la investigación ética y la física tienen, respectivamente, exigencias diferentes (que es, creo, lo único que defendería Rawls) es, cuanto menos, una caracterización sumamente laxa, e incluso incorrecta si se piensa que Platón defendió la tesis de la unidad de los saberes. Pero al margen de estas consideraciones, en estos momentos hasta cierto punto secundarias, lo que interesa ahora poner de manifiesto es que, para Nussbaum, el anti-platonismo no implica relativismo, como sostiene la otra concepción rival del modelo terapeútico, esto es, la concepción que toma pie en la creencia ordinaria. Así, el modelo terapéutico (tras el fracaso del aristotelismo) se presentaría como una especie de "tercera vía" entre el platonismo y el relativismo. Sin embargo, ya hemos visto que no consigue separarse claramente del platonismo, pues o bien cae en posiciones insalvablemente relativistas o bien, para evitar tal relativismo, tiene que renovar tesis platónicas.

La concepción que toma pie en la creencia ordinaria sostiene que la investigación ética es, simplemente, la transposición de las creencias vigentes en cada momento y en cada sociedad y que no hay ningún fin legítimo por detrás de éste. Frente a esta concepción, Nussbaum recuerda que las éticas helenistas sí que mantienen una idea normativa de qué es una vida buena; pero esta normatividad no hay que entenderla al modo platónico. El filósofo-médico (como si fuera una especie de «intelectual flotante" a la manera de Mannheim) tiene autoridad, pero que no es como la autoridad platónica, puesto que pertenece a la misma comunidad que el paciente, sólo que representando un ideal al que aspira este último ${ }^{58}$. Pero el problema no es éste, sino saber en virtud de qué tiene el filósofo-médico esta autoridad y en virtud de qué puede servir como ideal a imitar. Descartada la hipótesis teológica que afirmaría que el filósofomédico es un ungido de los dioses, sólo cabe responder que esta autoridad descansa en la posesión de un saber, que convierte al que lo posee en punto de referencia. No hay, pues, como en el platonismo que se inventa Nussbaum un

57 Cfr. pág. DA SILVA y W. NORMAN, «Rawlsianismo metodológico. Una introducción a la metodologia dominante en la filosofia política anglosajonan, en Revista Internacional de Filosofia Politica, 5, 1995, págs. 125-151.

58 Cfr. The Theraphy of..., pág. 27. 
conjunto de normas éticas independendientes de los deseos y formas de vida de los seres humanos; no hay, tampoco, relativismo, pero porque las normas éticas se han encarnado en una persona concreta (que puede llegar a ser venerada como un dios, como sucedió con Epicuro).

Tal era, en último extremo, la solución aristotélica: hay que hacer lo que dice el "varon prudente" porque este kanón kaì metrón. ¿Pero qué sucede entonces con las exigencias de libertad y autonomía a las que, como se ha señalado en páginas anteriores, Nussbaum no quiere renunciar? Sucede que no tiene cabida en el modelo terapéutico, debido a la esencial asimetría que este postula entre médico y paciente: este modelo sólo funciona cuando a la competencia del filósofo-médico se une la voluntad de curación por parte del paciente-discípulo, pero no bajo las condiciones de enfrentamiento estratégico. Ahora bien, es en esta última circunstancia cuando surje el problema que debe solucionar la filosofia práctica, pues en la primera es que, sencillamente, no hay problema que solucionar: sólo en este caso la "tercera vía" de Nussbaum funciona como tal "tercera vía", esto es, cuando lo hace en el vacío. 